\title{
Review Article \\ Mindfulness-Based Therapies in the Treatment of Functional Gastrointestinal Disorders: A Meta-Analysis
}

\author{
Monique Aucoin, Marie-Jasmine Lalonde-Parsi, and Kieran Cooley \\ Canadian College of Naturopathic Medicine, 1255 Sheppard Ave East, Toronto, ON, Canada M2K 1E2 \\ Correspondence should be addressed to Monique Aucoin; maucoin@ccnm.edu
}

Received 4 July 2014; Accepted 19 August 2014; Published 11 September 2014

Academic Editor: Toku Takahashi

Copyright ( 2014 Monique Aucoin et al. This is an open access article distributed under the Creative Commons Attribution License, which permits unrestricted use, distribution, and reproduction in any medium, provided the original work is properly cited.

Background. Functional gastrointestinal disorders are highly prevalent and standard treatments are often unsatisfactory. Mindfulness-based therapy has shown benefit in conditions including chronic pain, mood, and somatization disorders. Objectives. To assess the quality and effectiveness reported in existing literature, we conducted a meta-analysis of mindfulness-based therapy in functional gastrointestinal disorders. Methods. Pubmed, EBSCO, and Cochrane databases were searched from inception to May 2014. Study inclusion criteria included randomized, controlled studies of adults using mindfulness-based therapy in the treatment of functional gastrointestinal disorders. Study quality was evaluated using the Cochrane risk of bias. Effect sizes were calculated and pooled to achieve a summary effect for the intervention on symptom severity and quality of life. Results. Of 119 records, eight articles, describing seven studies, met inclusion criteria. In six studies, significant improvements were achieved or maintained at the end of intervention or follow-up time points. The studies had an unclear or high risk of bias. Pooled effects were statistically significant for IBS severity $(0.59,95 \%$ CI 0.33 to 0.86$)$ and quality of life $(0.56,95 \%$ CI 0.47 to 0.79$)$. Conclusion. Studies suggest that mindfulness based interventions may provide benefit in functional gastrointestinal disorders; however, substantial improvements in methodological quality and reporting are needed.

\section{Introduction}

Functional gastrointestinal disorders (FGIDs) have a high prevalence, a significant impact on patients' wellbeing and are costly to the health care system [1]. Patients with these disorders report a marked impact on quality of life and an average of 30 sick days per year per person, constituting a substantial health care burden [2].

The pathophysiology underlying FGIDs is unclear as they lack any discernable organic or structural pathology. Current knowledge suggests the involvement of factors such as abnormal gut motor function, increased visceral perception, abnormalities in central pain processing, and disruption of the gut microbiota as well as genetic and psychological factors [1]. Psychiatric disorders are frequent comorbidities in patients with FGIDs and recent prospective study evidence suggests that the relationship is bidirectional [1].

Of the FGIDs, the most common is irritable bowel syndrome (IBS), affecting $7-10 \%$ of the population worldwide. It is characterized by recurring abdominal pain or discomfort and diarrhea or constipation [1].

Standard treatment for IBS is targeted at symptom control through the use of laxatives, antidiarrheal agents, antispasmodics, and antidepressant medications. Studies report that less than $50 \%$ of patients with IBS are satisfied with the standard medical treatment and many turn to alternatives. Studies of complementary and alternative medicine use in IBS populations have reported rates of $21-51 \%$ [2].

Treatment and burden of other FGIDs such as functional abdominal pain, vomiting, and dyspepsia are less well understood, although there is considerable categorical overlap with IBS. Similarly to IBS, other FGIDs are associated with high rates of complementary and alternative medicine usage. Pharmacological treatments for other FGIDs, aimed at targeting receptors with enteric and central nervous system effects, are similarly in the early stages of development [3-5].

Because of the significant involvement of emotional, cognitive, and neurological factors in IBS, a number of 
studies have investigated psychological interventions including cognitive behavioural therapy (CBT), hypnotherapy, and relaxation exercises. An early review suggested that all of these interventions have shown benefit [2].

A more recent addition to this list of interventions is mindfulness-based therapy (MBT), a form of psychotherapeutic treatment which uses meditation practices to assist patients in the cultivation of nonjudgemental awareness of the present moment. This involves monitoring of cognition, emotion, perception, and sensations and the development of nonreactivity to difficult or negative aspects of these experiences [6]. The use of mindfulness as a therapeutic tool began in the late 1970s with the development of the mindfulness-based stress reduction (MBSR) program as a treatment for chronic pain [7]. The MBSR program has been combined with CBT in the development of mindfulnessbased cognitive therapy (MBCT). It was developed for the prevention of major depressive disorder relapse [7], however evidence to support its use in anxiety and active depression continues to emerge [8]. The programs typically consist of 8 weekly 2.5 hour group sessions involving various forms of meditation, group discussion, and other exercises; one day of meditation retreat and approximately one hour of daily home practice [9].

In addition to the treatment of mental health concerns, there is an expanding body of research supporting the use of mindfulness-based interventions for stress, pain, and somatization disorders such as fibromyalgia and chronic fatigue syndrome [7].

A review article exploring the neural mechanisms of mindfulness and meditation found significant structural and functional changes within the brain both during, and resulting from, mindful states and practices [10]. Based on patterns of cortical thickening, meditation is associated with structural changes in brain regions related to sensory, cognitive, and emotional processing [11].

Because of the significant involvement of emotional factors in IBS, it was initially suspected that the benefit of psychological interventions was achieved through improvement of comorbid psychological distress [6]. A recent study utilized a number of assessment tools to explore some hypothesized mechanisms for the benefit exerted by MBT on IBS. The results of their analysis revealed that several cognitive processes are involved. MBT led to a decrease in reactivity to thoughts, emotions and physical sensations which led to a decrease in visceral sensitivity. The decreased visceral sensitivity was related to a decrease in IBS symptom severity and an improvement in quality of life. Additionally, nonreactivity was associated with a decrease in pain catastrophizing which predicts improvement in quality of life and increased reinterpretation of pain sensations predicted reductions in IBS severity [6].

Previous reviews studying the use of MBT in FGIDs have combined it with other psychotherapeutic interventions or with other disorders $[7,12]$. A recent systematic review and meta-analysis investigated the use of mindfulness-based therapy in the treatment of somatization disorders including fibromyalgia, chronic fatigue, and IBS [7]. In the time since this review was completed, additional clinical trials have been published. The review examined efficacy outcomes at the end of treatment only and did not discuss risk of bias or other elements describing the quality of reporting of the studies. A synthesis which includes these components is essential to provide context to the findings as well as provide guidance for future research.

This review will discuss the effectiveness of mindfulness therapy at improving symptom severity and quality of life measures in patients diagnosed with FGIDs compared to waitlist or active control groups. The review will explore the effectiveness at the end of the intervention as well as after a follow-up period. Additionally, the quality of the studies will be assessed to describe the current state of reporting and study bias in the existing literature.

\section{Methods}

The PRISMA statement was used to guide the conduct and reporting of this meta-analysis [13].

2.1. Systematic Literature Searches. Systematic literature searches were performed using the Pubmed, EBSCO, and Cochrane databases. The following search terms were used: mindfulness, MBCT, MBSR, mindfulness-based cognitive therapy, mindfulness-based stress reduction, mindful, functional gastrointestinal, functional bowel, colonic disease functional, colonic disease, functional abdominal pain, recurrent abdominal pain, abdominal pain, IBS, irritable bowel, spastic colon, irritable colon, constipation, diarrhea, bloating, distention, gastroesophageal reflux, GERD, dysphagia, and functional dyspepsia. Studies in any stage of publication from database inception onward in English were considered. The purpose of this strategy was to be inclusive of the existing literature and noting that previous reviews did not identify a large base of non-English publications. The last date searched was May 29, 2014.

The search results were combined and duplicates were removed. A screen of article titles and abstracts was performed to identify clinical trials that utilized mindfulnessbased interventions for the treatment of FGIDs. After reviewing the full-text articles, those with control groups, randomization, and an adult population with FGID symptoms were included.

2.2. Data Collection. Data was extracted by one reviewer. Data for the following study variables was extracted: study size and percent female participants, participant diagnosis, intervention and duration, control, follow-up, symptom severity at the end of the intervention and at follow-up, and quality of life assessment at the end of the intervention and at follow-up. The principle summary outcome measures for synthesis were the changes in symptoms severity between baseline, end-of-intervention, and follow-up. Corresponding authors of included studies were contacted regarding missing or unclear data, though notably this did not result in any additional information beyond what was originally published. Two attempts to contact authors via email were made before ceasing attempts at correspondence. 
2.3. Data Analysis. Effect sizes (Cohen's $d$ ) were calculated for relevant validated outcome measures (effect on IBS severity at end of intervention, effect at postintervention follow-up, and quality of life) from individual studies using reported mean, standard deviation and group size. A random effects model (DerSimonian-Laird (DL)) was assumed to account for the small number of studies with pool-able data $(n=5-6)$, small sample sizes, and high degree of variance within the studies. Studies were weighted based on sample size in order to generate a pooled point estimate and $95 \%$ confidence interval for effect size. Heterogeneity was assessed using the $I^{2}$ statistic; Cochran $Q$ is reported as an inference of combinability of studies. Kendall's tau and Egger's test will be reported to assess for power and risk of bias affecting the cumulative result. Statistical analysis and figure generation (funnel and forest plots) were accomplished using StatsDirect (version 3.0.119) software.

2.4. Quality Analysis. Assessment of study quality was conducted using the Cochrane Risk of Bias [22] and the CONSORT checklist for reporting trials of nonpharmacologic treatments [23]. Assessment was completed by two reviewers independently and any discrepancies were discussed until a consensus was reached.

\section{Results}

3.1. Literature Search. The literature search yielded 119 unique records (Figure 1). After these records were screened based on title and abstract, 106 studies were excluded. The reasons included the following: did not assess the use of mindfulness in FGIDs (85), review articles (14), protocol only (2), uncontrolled design (1), pediatric population (1), other types of pain included (1), outcomes limited to cost effectiveness (1), and outcomes limited to psychological symptoms (1). Of the 13 full-text articles assessed for eligibility, eight articles reporting the results of seven randomized controlled trials met the criteria for inclusion in this analysis. The reasons for exclusion were a lack of adequate control (1), combination with other somatic disorders (1), not written in English (1), only mechanism of action outcomes reported (1), and reporting the same results as another included study (1).

3.2. Efficacy-End of Intervention. Of the seven studies included in this review, five (71.4\%) reported significant improvements in IBS symptom severity at the end of the intervention compared to waitlist or comparison intervention (Table 1). One study did not report end-of-intervention results [24]. One study, which included patients with inflammatory bowel disease (IBD) who were in remission and experiencing IBS-like symptoms, showed a nonsignificant trend towards improvement compared to waitlist control. These patients represented a subgroup analysis within the study and, thus, had a small sample size [14].

3.3. Efficacy-Follow-Up. Data from a follow-up time point was reported in all eight publications. These follow-up periods ranged from two to 18 months after the end of the intervention. The study of IBD patients continued to show a

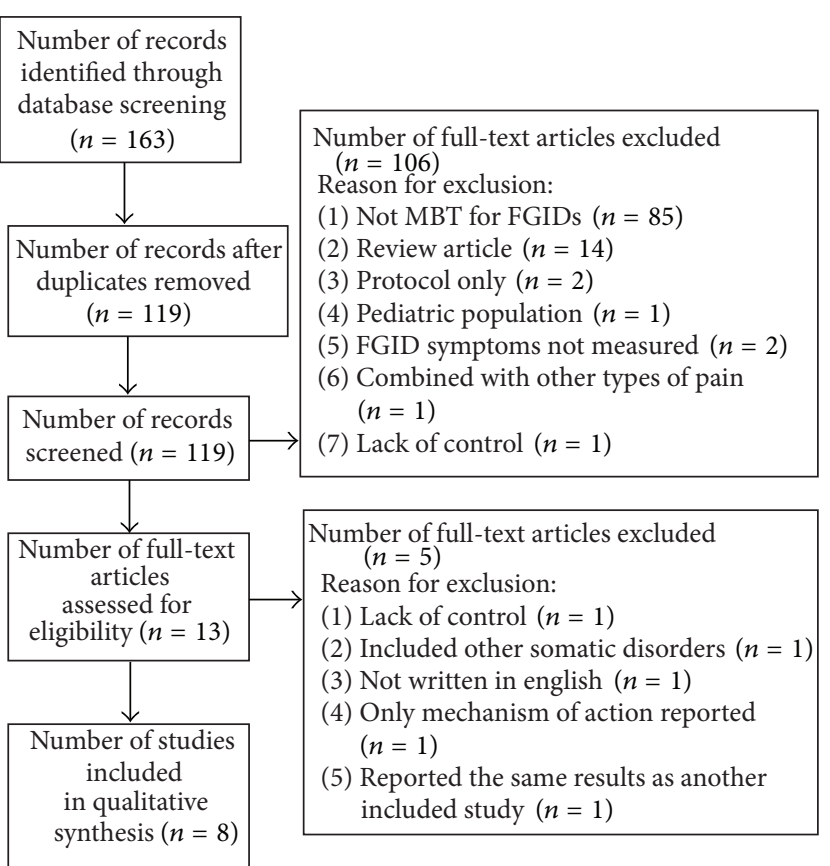

FIGURE 1: PRISMA flow chart showing number of screened, included, and excluded studies.

trend towards improvement that did not reach significance [14]. The study that only reported data from the follow-up assessment showed significant improvement [21]. The other six studies reported that participants maintained improvement in the severity of their IBS symptoms. Among these, one showed a nonsignificant trend towards further improvement [19]. One study that showed maintenance of improvement showed improvement in the control group resulting in a loss of statistical significance [20]. During the follow-up period the participants did not receive further treatment with mindfulness-based therapy; however the programs taught participants skills and exercises which they were encouraged to continue using. Two studies assessed for the use of additional treatments during the follow-up period and found no significant difference in the outcomes reported by those who had sought additional treatment and those who had not $[17,18]$.

3.4. Efficacy-Quality of Life. Five studies utilized the irritable bowel syndrome quality of life instrument (IBS-QOL) as a secondary outcome and of these, $80.0 \%(n=4)$ reported a significant improvement at end-of-intervention. Between the end-of-intervention and the follow-up assessment, significant further improvement was seen in two of these studies while the other two studies showed maintenance of improvement. One study demonstrated a significant improvement in IBS-QOL in both the intervention group and the wait list control group that was maintained at follow-up [20]. The study reporting long-term follow-up data only showed maintenance of QOL improvement.

The study that enrolled IBD patients used an objective biomarker for the assessment of intestinal inflammation [14]; 


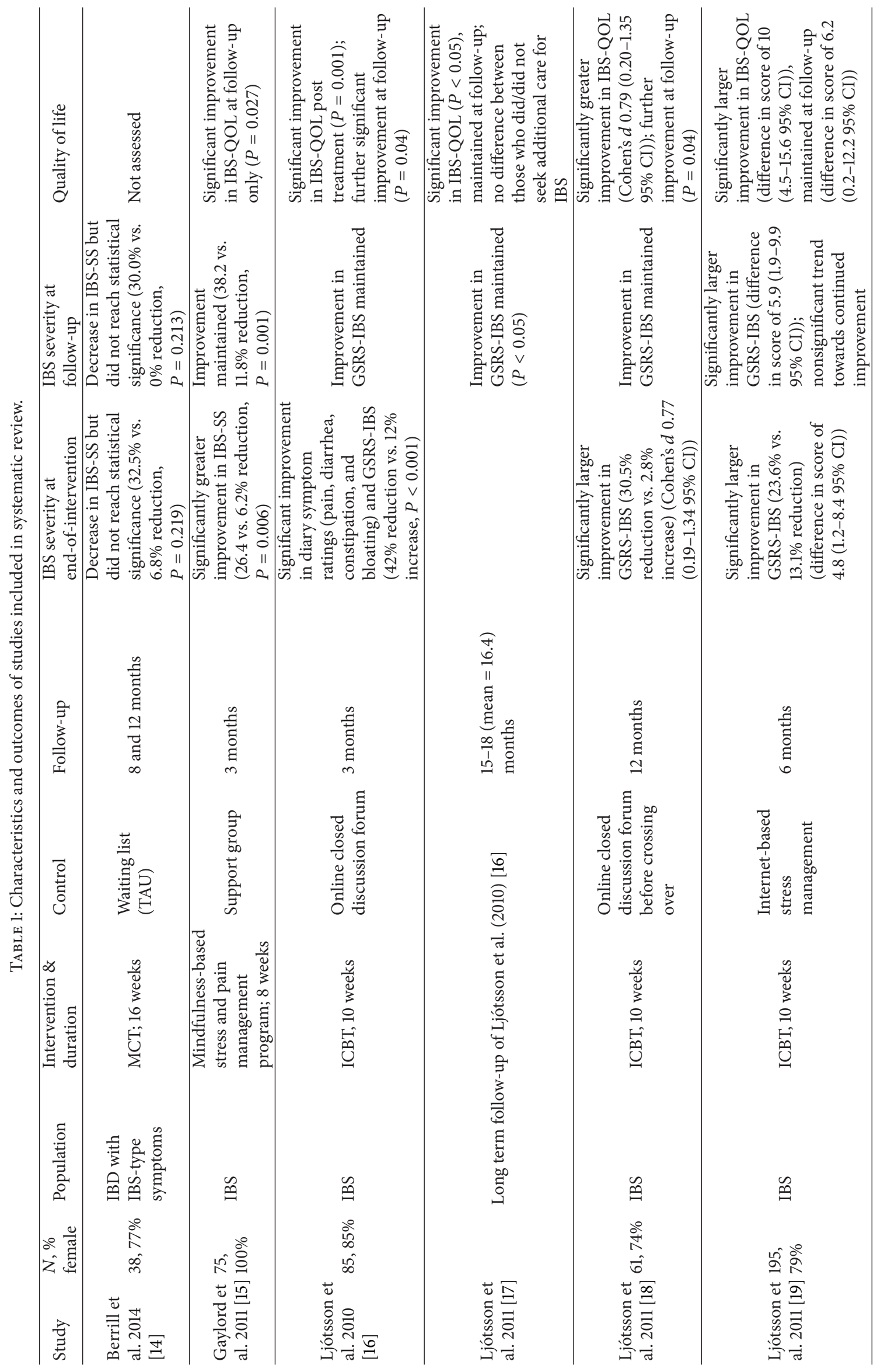




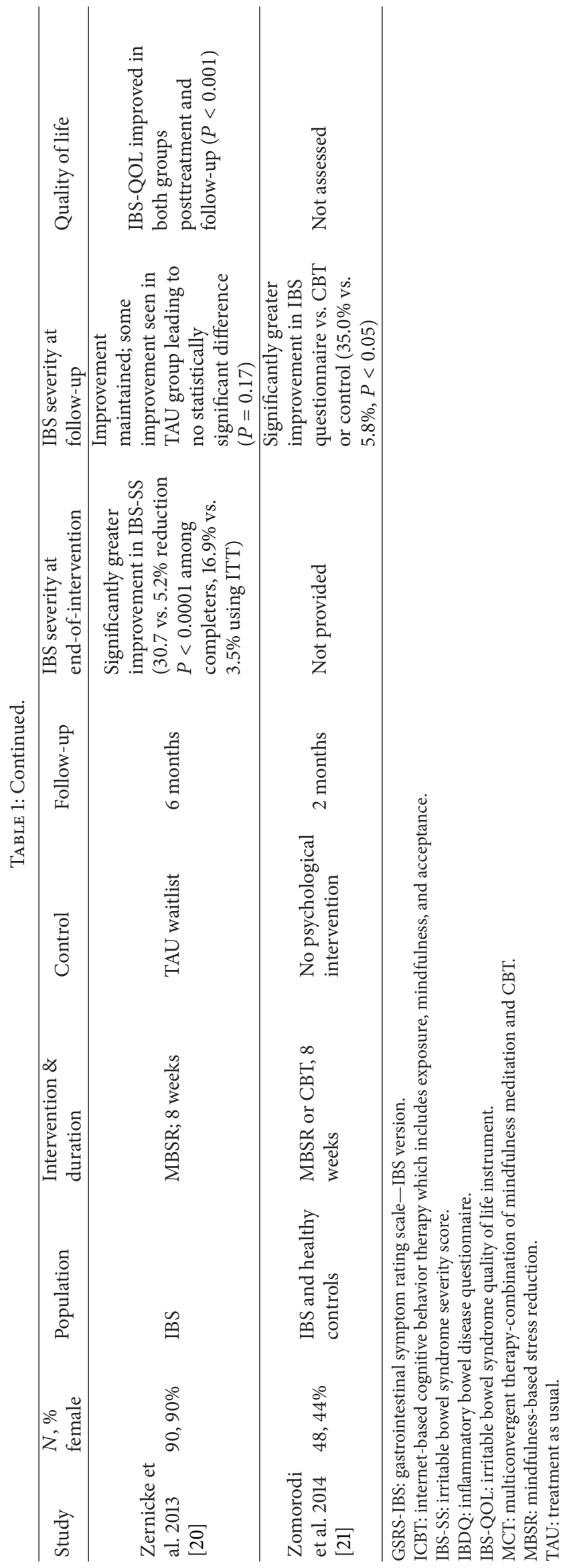


however none of the other studies used objective tests for the assessment of FGID symptoms as primary or secondary outcome measures. All of the assessment tools relied on validated patient/self-report outcome measures.

Two studies $[18,19]$ used a linear mixed-effects model to observe the difference in rates of change between the MBT and control intervention over time amid significant interaction effects between group and time were seen $(P<$ $001)$.

3.5. Quality Assessment. Quality assessment of the studies included in the review revealed strengths as well as weaknesses and opportunities for the introduction of bias. The Cochrane risk of bias assessment showed overall unclear or high risk of bias for the included studies (Table 2).

The most significant contributor to risk of bias was a lack of blinding of participants, facilitators, and outcome assessment. In three studies, the mindfulness intervention was compared with a support group or another psychological intervention and the participants were not aware of their allocation in the study; however, the remaining studies used a waitlist control or treatment-as-usual comparison and in these cases, the participants were aware that they were receiving the intervention being tested. In all studies, personnel who were administering the therapy were not blinded, although this is acknowledged as an inherent challenge in psychological interventions.

Another area that presented a risk of bias is incomplete outcome data. In many studies the rate of withdrawal was the same in the intervention and control groups and intention to treat analyses were utilized; however, in many cases the dropout rates were large, ranging from 10 to $44 \%$. One study failed to report outcome measures at the end of the intervention and only reported data from the follow-up assessment. Two studies failed to describe their funding source. Some studies lacked clarity in their description of random sequence generation $(n=1)$ and allocation concealment $(n=3)$.

Assessment of the studies using the CONSORT checklist of items for reporting trials of nonpharmacologic treatment also highlighted strengths and weaknesses (Figure 2). The majority of studies included adequately reported background information, study objectives, sample size determination, randomization method, statistical analysis methods, participant flow, recruitment dates, baseline data, numbers analyzed, outcomes, additional analyses, interpretations, generalizability, and overall evidence. Partially complete information was reported in most titles and abstracts. There was limited reporting of the inclusion criteria for study sites and intervention providers as well as the location of data collection. Additionally, only two studies completely described standardization of the intervention and assessment of adherence to the protocol. None of the studies reported adverse event data or results of how the interventions were implemented. As previously stated, the details of allocation concealment were often incomplete or absent, as well as information about blinding of participants and personnel. Of the eight studies, four reported registration in an open access clinical trial registry.

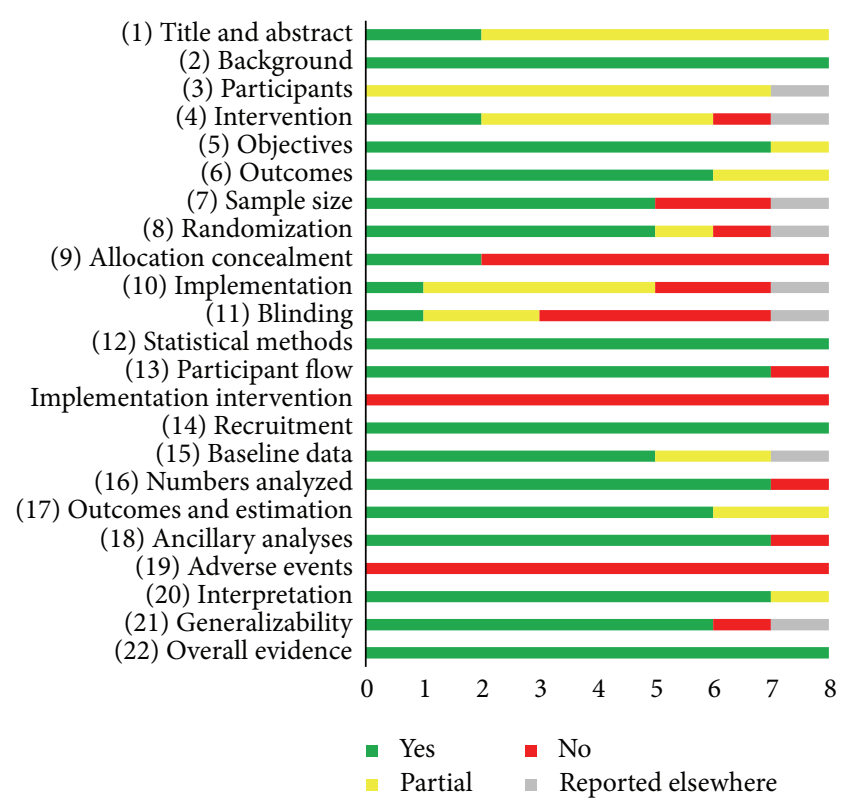

FIGURE 2: CONSORT checklist of items for reporting trials of nonpharmacologic treatments.

Overall, the studies included had deficiencies in reporting and significant risk of influence of bias.

3.6. Meta-Analysis. Six studies reported IBS severity at end of intervention data that was amenable to calculation of effect size; five studies contained data available for pooling for each of IBS severity at postintervention follow-up and quality of life.

Mild-moderate heterogeneity existed between studies with respect to effects of $\mathrm{MBT}$ on IBS severity at end of intervention $\left(I^{2}=49.9 \%, 95 \% \mathrm{CI}=0 \%\right.$ to $78.2 \%$; Cochran $Q=$ 9.982 $P=0.076)$, on IBS severity at postintervention followup $\left(I^{2}=23.3 \%, 95 \% \mathrm{CI}=0 \%\right.$ to $71.8 \%$; Cochran $Q=5.216$ $P=0.266)$, and on QOL $\left(I^{2}=30.4 \%, 95 \% \mathrm{CI}=0 \%\right.$ to $74 \%$; Cochran $Q=5.747 P=0.219)$.

Funnel plots (Figure 3), Kendall's tau, and Egger's test for bias are suggestive of low power, low likelihood for unpublished or unreported studies, and not statistically significant for bias across IBS severity at end-of-intervention, (Kendall's tau $=0.333 P=0.469$; Egger $=1.901,95 \% \mathrm{CI}=-4.376$ to 8.182 $P=0.448$ ), on IBS severity at postintervention follow-up (Kendall's tau $=0.4 P=0.483$; Egger $=1.256,95 \% \mathrm{CI}=-3.988$ to 6.501, $P=0.501$ ), and on QOL (Kendall's tau $=0 P=0.817$; Egger $=1.345,95 \% \mathrm{CI}=-6.742$ to $9.432, P=0.633)$.

Forest plots (Figure 4) outline a statistically significant pooled effect size for IBS severity at end of intervention (Pooled $d=0.596,95 \% \mathrm{CI}=0.334$ to 0.858 ), on IBS severity at postintervention follow-up (Pooled $d=0.352,95 \% \mathrm{CI}=0.112$ to 0.593 ), and on QOL (Pooled $d=0.564,95 \% \mathrm{CI}=0.340$ to 0.789 ) using random effects model. No major difference in findings was observed using a fixed effects model for pooling data (data not reported). 


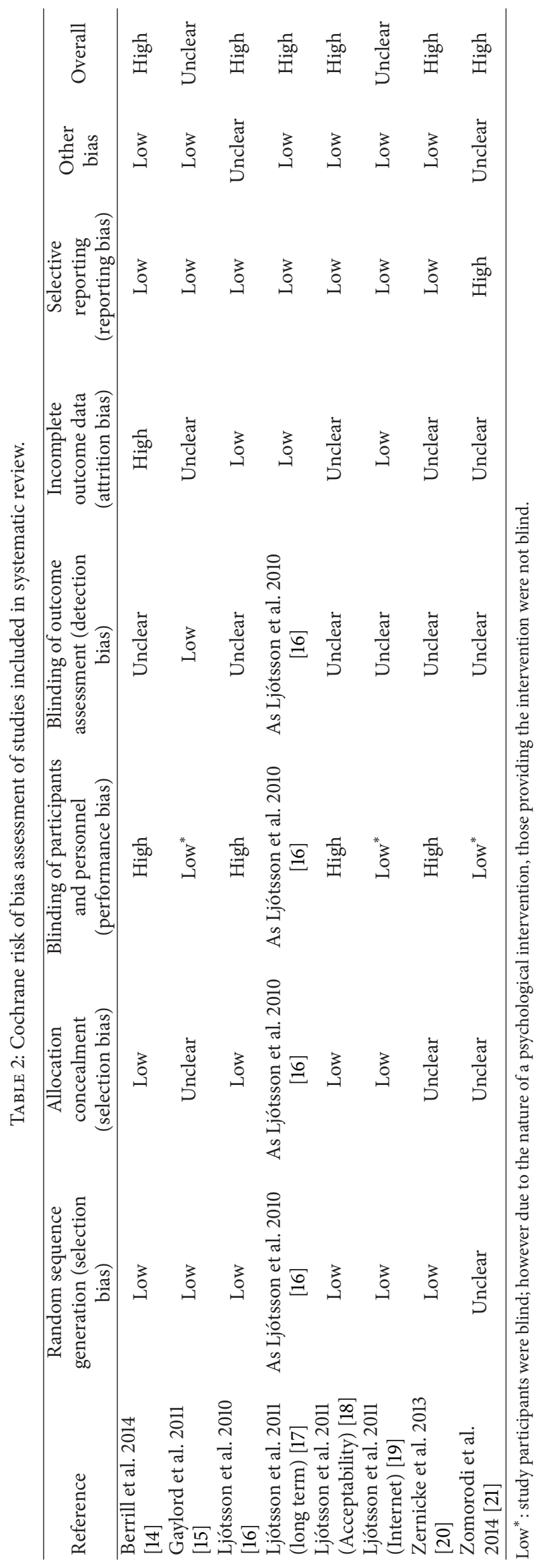




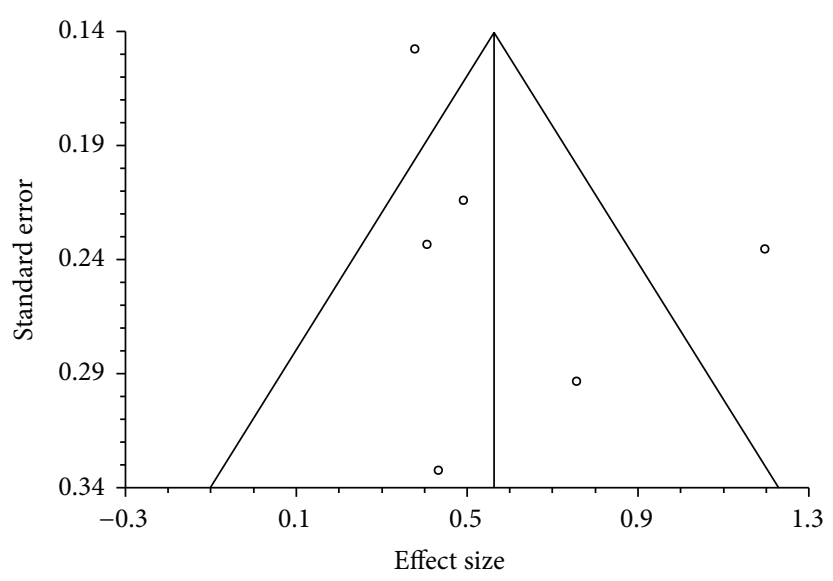

(a)

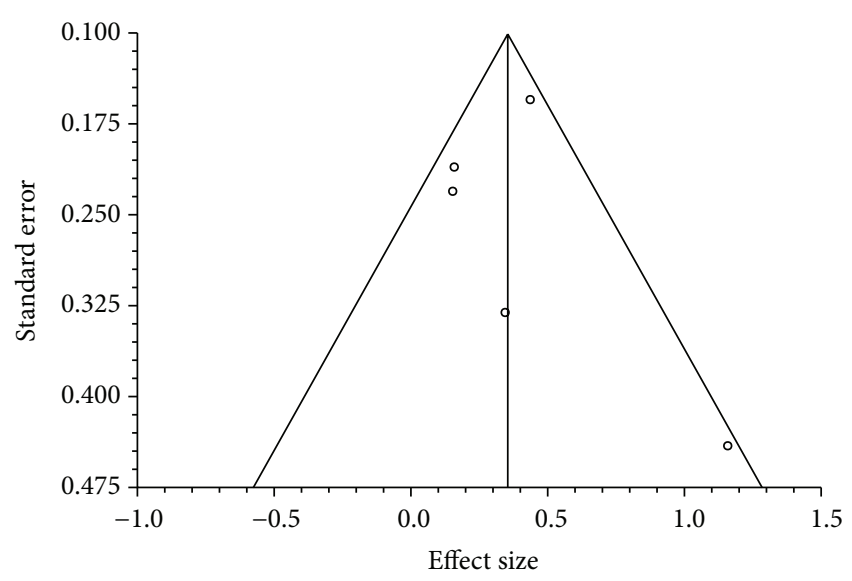

(b)

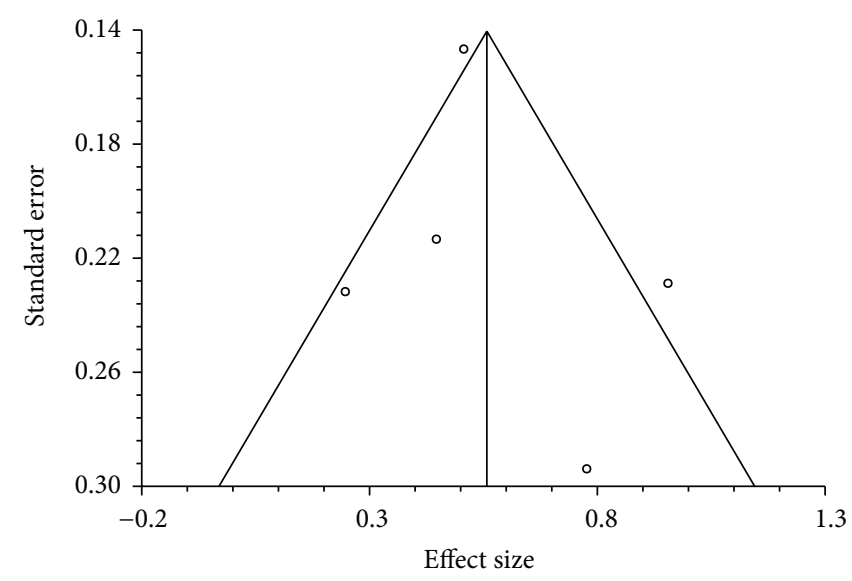

(c)

FIGURE 3: Funnel plots for IBS severity at end of intervention (a), IBS severity at postintervention follow-up (b), and quality of life (c).

\section{Discussion}

The results of the studies reviewed suggest that MBT may be an effective treatment for FGIDs achieving both a reduction of symptom severity and an improvement in quality of life. The mean decrease in symptom severity ranged from 23 to $42 \%$. Though the sample size is small, this suggests some consistency in effectiveness observed amongst studies. A previous meta-analysis suggests that the variability of effectiveness of mindfulness therapies is no greater than that observed in other pharmacological or cognitive behavioural therapies across disorders [24]. In Zernicke et al. [20], the mean decrease of $30.7 \%$ amongst completers equated to $50 \%$ of participants achieving a clinically meaningful reduction in their IBS symptoms (i.e., a reduction of 50 points on the IBS Severity Scale).

4.1. Duration of Effect. Additionally, the results suggest that the improvement achieved during treatment is lasting and may even lead to continued improvement. All of the studies that yielded statistically significant improvement in symptom severity at end-of-intervention demonstrated maintenance of that improvement at follow-up. In addition, three studies observed statistically significant improvement in quality of life between end-of-intervention and follow-up. Lasting effects have been observed in previous studies using MBT. One study, which sought to investigate the long-term effects of MBCT in the treatment of depression, found that improvements achieved during treatment were maintained for up to 59.8 months of follow-up [25]. The lasting effects of MBT are likely related to changes in the way participants attend to moment-by-moment cognition, emotion, perception, and sensations - the development of trait or dispositional mindfulness [6].

4.2. Quality. Quality assessment of the studies revealed some strengths, but largely weaknesses and deficiencies. Overall, the current literature has not responded to challenges relating to increased quality in design, conduct, and reporting that may impact credibility in the field of mindfulness or other psychological interventions [26].

Some of the studies used active control groups including support groups, discussion forums, cognitive behavioral therapy, and stress-management training. This allowed for 


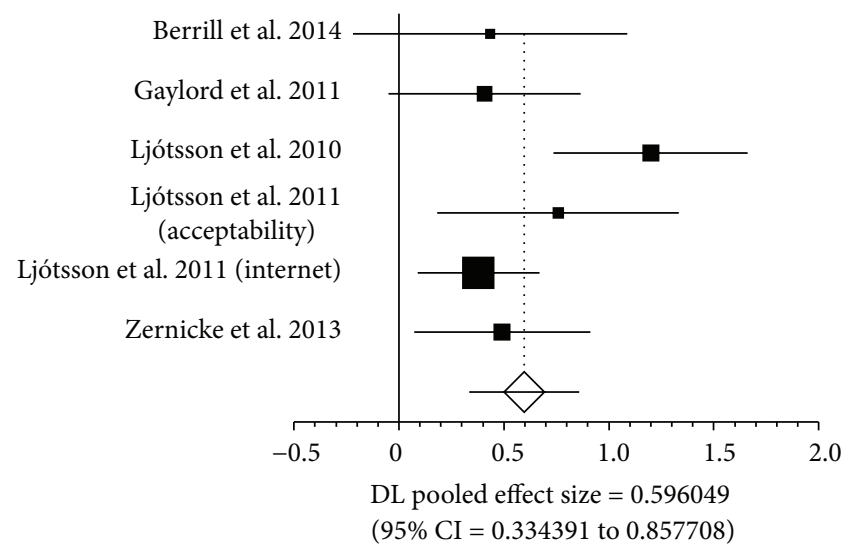

(a)

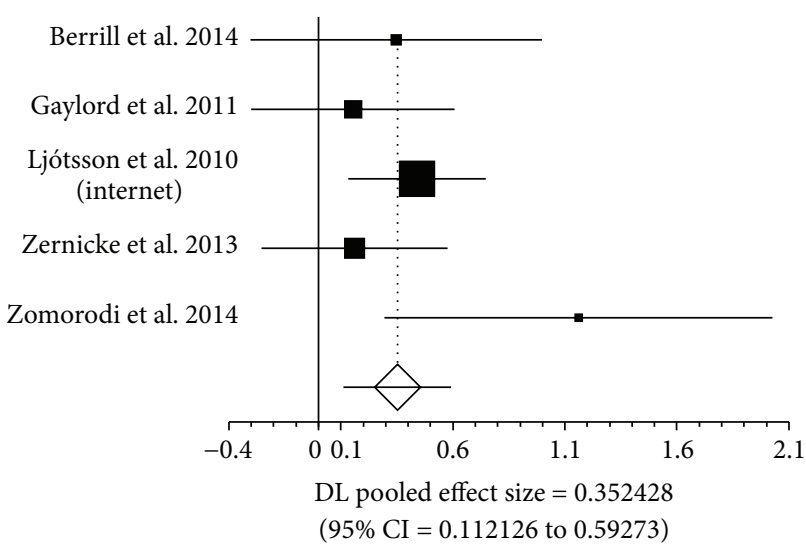

(b)

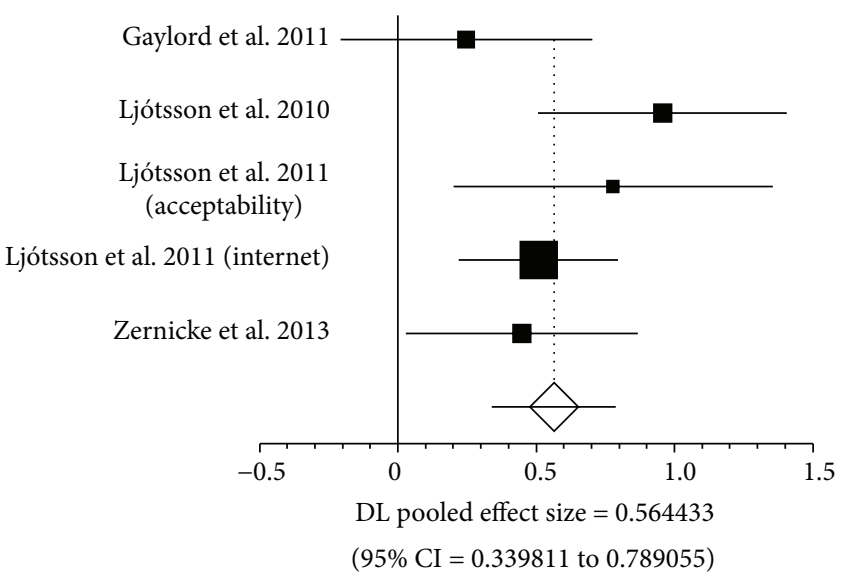

(c)

FIGURE 4: Forest plots for effect size on IBS Severity at end of intervention (a), IBS severity at postintervention follow-up (b), and quality of life (c).

participant blinding as well as insight into the mechanism of the effect. In all cases the mindfulness based therapy showed superior efficacy to the other interventions suggesting that the therapeutic benefit is specific to the material covered rather than nonspecific factors such as peer-support, attention, or the expectation effect. However, a major challenge in the study of psychological interventions is the inability to blind all study personnel to participant allocation. Some studies took steps to help conceal allocation and preserve blinding amongst outcome assessors; however no studies took into account blinding of the individuals facilitating the interventions or other steps that might help manage expectation and performance bias.

Another area that posed a risk of bias is incomplete outcome data due to dropouts. MBT requires a large amount of participant involvement and time, often including weekly group sessions and daily home practice. This may have contributed to the high dropout rates observed. Many studies utilized intention to treat analysis to account for these occurrences however some articles did not address this or report the specific manner in which intention to treat analysis was done.
A major limitation to this review is a relatively small number of studies with (qualitatively) significant heterogeneity in their methodology. The follow-up time period varied from two to 18 months. Additionally, the type of intervention varied. Of the seven studies reviewed, three were conducted by the same research group using a unique methodology called internet-based cognitive behaviour therapy (ICBT) which includes mindfulness and acceptance-based exercises in combination with exposure. While it is accessible over the internet, it is not available to the public at this time. In contrast, MBSR and MBCT programs are offered in hospitals, universities and health clinics worldwide.

Most of the studies reviewed enrolled patients with a diagnosis of IBS. The one study that included participants with IBD in remission and IBS-like symptoms was the only study that failed to yield a statistically significant improvement in IBS symptoms. The patients with IBS-type symptoms in this study were a subset of a larger patient population and as a result there was a small sample size which may have contributed to the failure to reach statistical significance. Alternatively, it may be that patients without organic gastrointestinal disease are more responsive to MBT. 
Many of the studies had a high percentage of female participants. While there is a risk that this may limit the generalizability of the results it is known that IBS is more prevalent among women [7].

The studies reviewed demonstrated benefits in the placebo groups; however, this is a common finding among trials involving patients with IBS and other subjective complaints. A meta-analysis of the placebo effect in IBS found a range of $16-71 \%$ improvement (27) and a randomized controlled trial using open-label placebo for the treatment of IBS demonstrated a statistically significant benefit (28).

Although a statistically significant finding was demonstrated on pooled effect sizes, the low power, small number of studies, and overall high risk of bias in study design or completeness of reporting suggest that this should be interpreted with some discretion.

\section{Conclusions}

Analysis of these studies suggests that mindfulness-based interventions may be useful in improving FGID symptom severity and quality of life with lasting effects; however, substantial improvements in methodological quality must be implemented in future studies in order to fully assess its impact. Due to absence of reporting of adverse events, no definitive conclusions can be drawn with respect to safety. Future studies would benefit from use of established criteria for reporting clinical trials using nonpharmacological interventions, registration of studies in an open-access clinical trial registry, and improvements in blinding to decrease the risk of bias.

\section{Conflict of Interests}

The authors declare that there is no conflict of interests regarding the publication of this paper.

\section{Acknowledgment}

Deborah Kennedy assisted in the development of the search strategy and paper editing.

\section{References}

[1] G. De Palma, S. M. Collins, and P. Bercik, "The microbiotagut-brain axis in functional gastrointestinal disorders," Gut Microbes, vol. 5, no. 3, 2014.

[2] D. J. Kearney and J. Brown-Chang, "Complementary and alternative medicine for IBS in adults: mind-body interventions," Nature Clinical Practice Gastroenterology \& Hepatology, vol. 5, pp. 624-636, 2008.

[3] M. Camilleri, "Novel therapeutic agents in neurogastroenterology: advances in the past year," Neurogastroenterology and Motility, vol. 26, no. 8, pp. 1070-1078, 2014.

[4] F. Cremonini, "Standardized herbal treatments on functional bowel disorders: moving from putative mechanisms of action to controlled clinical trials," Neurogastroenterology \& Motility, vol. 26, no. 7, pp. 893-900, 2014.
[5] F. Jing and J. Zhang, "Metabolic kinetics of 5-hydroxytryptamine and the research targets of functional gastrointestinal disorders," Digestive Diseases and Sciences, 2014.

[6] E. L. Garland, S. A. Gaylord, O. Palsson, K. Faurot, J. Douglas Mann, and W. E. Whitehead, "Therapeutic mechanisms of a mindfulness-based treatment for IBS: effects on visceral sensitivity, catastrophizing, and affective processing of pain sensations," Journal of Behavioral Medicine, vol. 35, no. 6, pp. 591-602, 2012.

[7] S. E. Lakhan and K. L. Schofield, "Mindfulness-based therapies in the treatment of somatization disorders: a systematic review and meta-analysis," PLoS ONE, vol. 8, no. 8, Article ID e71834, 2013.

[8] A. Chiesa and A. Serretti, "Mindfulness based cognitive therapy for psychiatric disorders: a systematic review and metaanalysis," Psychiatry Research, vol. 187, no. 3, pp. 441-453, 2011.

[9] M. Sharma and S. E. Rush, "Mindfulness-based stress reduction as a stress management intervention for healthy individuals: a systematic review," Journal of Evidence-Based Complementary \& Alternative Medicine. In press.

[10] W. R. Marchand, "Neural mechanisms of mindfulness and meditation: evidence from neuroimaging studies," World Journal of Radiology, vol. 6, no. 7, pp. 471-479, 2014.

[11] S. W. Lazar, C. E. Kerr, R. H. Wasserman et al., "Meditation experience is associated with increased cortical thickness," NeuroReport, vol. 16, no. 17, pp. 1893-1897, 2005.

[12] F. Asare, S. Störsrud, and M. Simrén, "Meditation over medication for irritable bowel syndrome? On exercise and alternative treatments for irritable bowel syndrome," Current Gastroenterology Reports, vol. 14, no. 4, pp. 283-289, 2012.

[13] D. Moher, A. Liberati, J. Tetzlaff, and D. G. Altman, "Preferred reporting items for systematic reviews and meta-analyses: the PRISMA statement," PLoS Medicine, vol. 6, no. 6, Article ID e1000097, 2009.

[14] W. Berrill, M. Sadlier, K. Hood, and J. T. Green, "Mindfulnessbased therapy for inflammatory bowel disease patients with functional abdominal symptoms or high perceived stress levels," Journal of Crohn's and Colitis, vol. 8, no. 9, pp. 945-955, 2014.

[15] S. A. Gaylord, O. S. Palsson, E. L. Garland et al., "Mindfulness training reduces the severity of irritable bowel syndrome in women: results of a randomized controlled trial," The American Journal of Gastroenterology, vol. 106, no. 9, pp. 1678-1688, 2011.

[16] B. Ljótsson, L. Falk, A. W. Vesterlund et al., "Internet-delivered exposure and mindfulness based therapy for irritable bowel syndrome-a randomized controlled trial," Behaviour Research and Therapy, vol. 48, no. 6, pp. 531-539, 2010.

[17] B. Ljótsson, E. Hedman, P. Lindfors et al., "Long-term followup of internet-delivered exposure and mindfulness based treatment for irritable bowel syndrome," Behaviour Research and Therapy, vol. 49, no. 1, pp. 58-61, 2011.

[18] B. Ljótsson, G. Andersson, E. Andersson et al., "Acceptability, effectiveness, and cost-effectiveness of internet-based exposure treatment for irritable bowel syndrome in a clinical sample: a randomized controlled trial," BMC Gastroenterology, vol. 11, article 110, 2011.

[19] B. Ljótsson, E. Hedman, E. Andersson et al., "Internet-delivered exposure-based treatment vs. Stress management for irritable bowel syndrome: a randomized trial," The American Journal of Gastroenterology, vol. 106, no. 8, pp. 1481-1491, 2011.

[20] K. A. Zernicke, T. S. Campbell, P. K. Blustein et al., "Mindfulness-based stress reduction for the treatment of irritable 
bowel syndrome symptoms: a randomized wait-list controlled trial," International Journal of Behavioral Medicine, vol. 20, no. 3, pp. 385-396, 2013.

[21] S. Zomorodi, S. Abdi, and S. K. Tabatabaee, "Comparison of long-term effects of cognitive-behavioral therapy versus mindfulness-based therapy on reduction of symptoms among patients suffering from irritable bowel syndrome," Gastroenterology and Hepatology from Bed to Bench, vol. 7, no. 2, pp. 118124, 2014.

[22] J. P. T. Higgins, D. G. Altman, P. C. Gøtzsche et al., "The Cochrane Collaboration's tool for assessing risk of bias in randomised trials," The British Medical Journal, vol. 343, no. 7829, Article ID d5928, 2011.

[23] I. Boutron, D. Moher, D. G. Altman, K. F. Schulz, and P. Ravaud, "Extending the CONSORT statement to randomized trials of nonpharmacologic treatment: explanation and elaboration," Annals of Internal Medicine, vol. 148, no. 4, pp. 295-309, 2008.

[24] B. Khoury, T. Lecomte, G. Fortin et al., "Mindfulness-based therapy: a comprehensive meta-analysis," Clinical Psychology Review, vol. 33, no. 6, pp. 763-771, 2013.

[25] K. Munshi, S. Eisendrath, and K. Delucchi, "Preliminary long-term follow-up of mindfulness-based cognitive therapyinduced remission of depression," Mindfulness, vol. 4, no. 4, pp. 354-361, 2013.

[26] J. C. Coyne, "Are most positive findings in health psychology false.... or at least somewhat exaggerated?" The European Health Psychologist, vol. 11, pp. 49-51, 2009. 


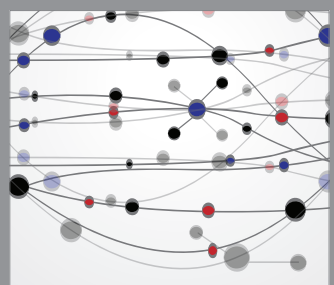

The Scientific World Journal
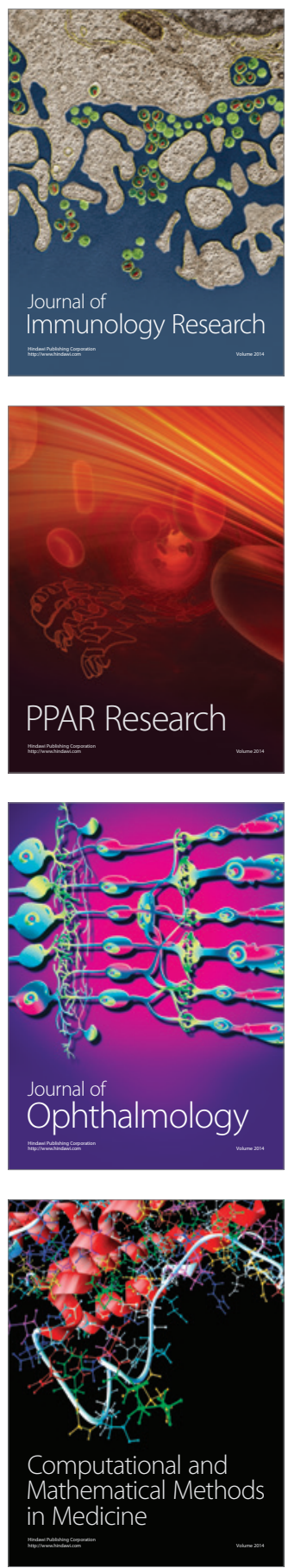

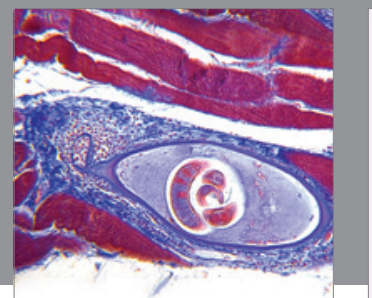

Gastroenterology

Research and Practice
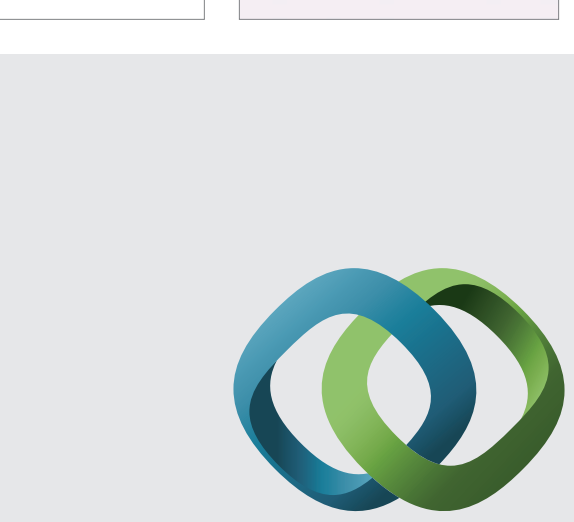

\section{Hindawi}

Submit your manuscripts at

http://www.hindawi.com
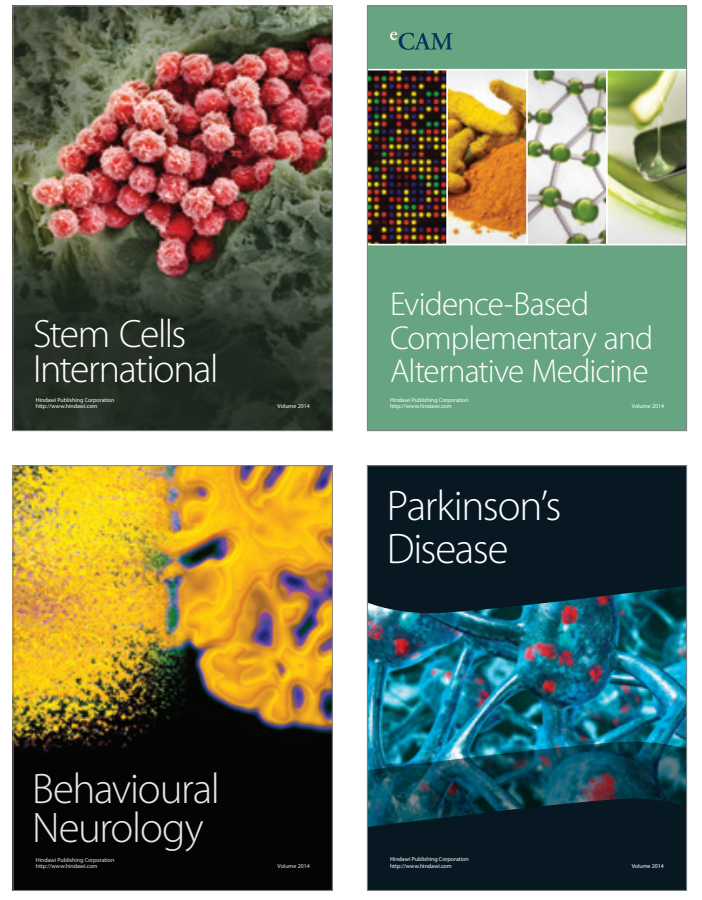
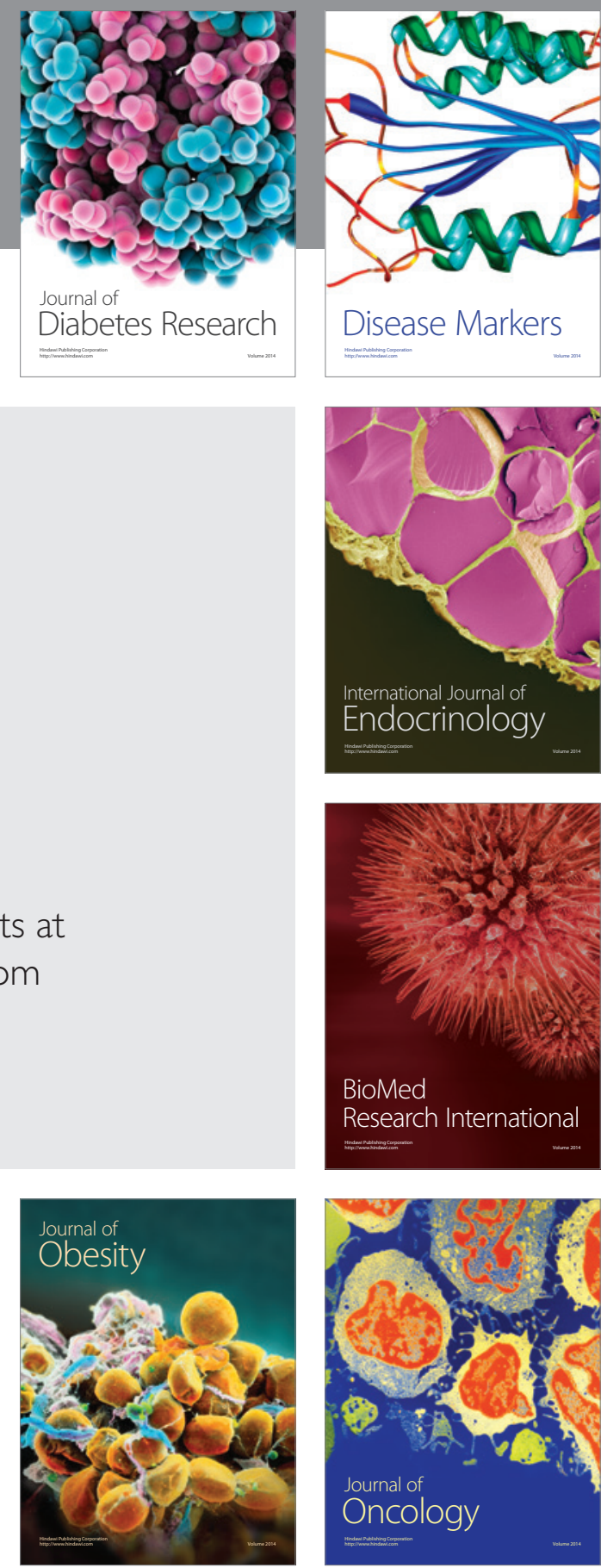

Disease Markers
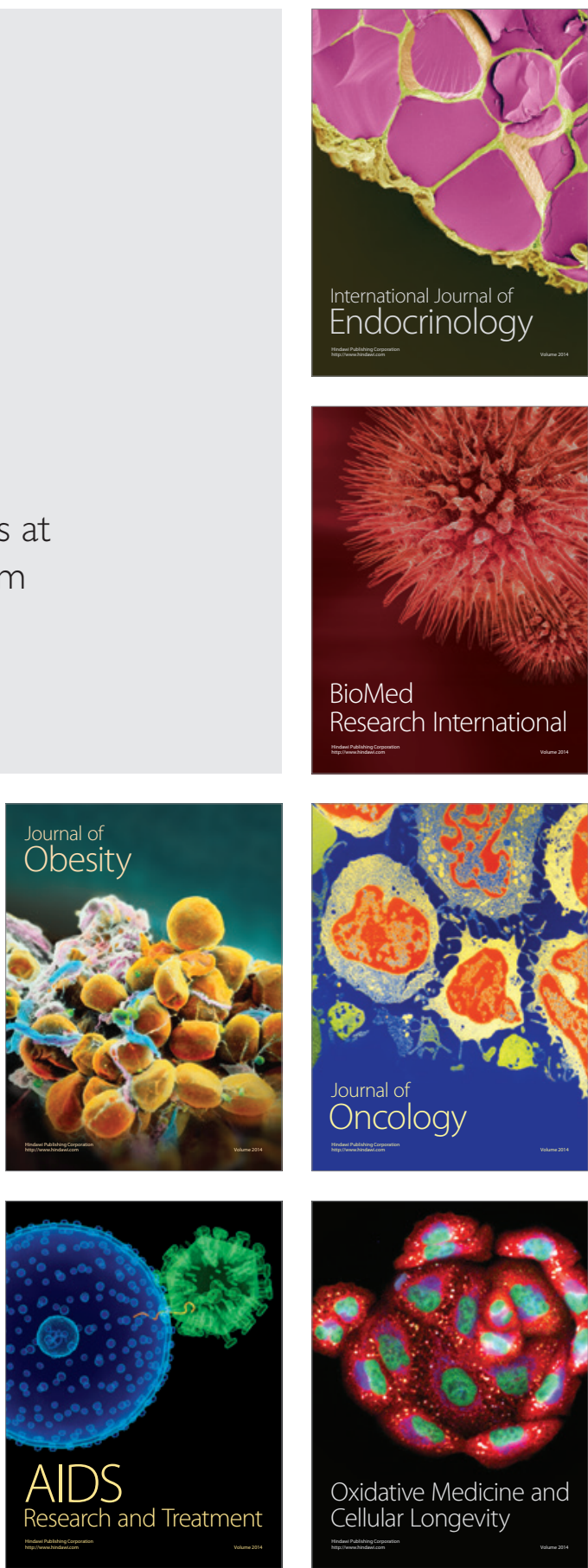